\title{
Rigorous approach to the problem of ultraviolet divergencies in dilute Bose gases
}

\author{
Maxim Olshanii1 (a) and Ludovic Pricoupenko'(b) \\ ${ }^{1}$ Department of Physics \& Astronomy, University of Southern California, Los Angeles, CA 90089-0484, USA \\ ${ }^{2}$ Laboratoire de Physique Théorique des Liquides, Université Pierre et Marie Curie, case 121, 4 place Jussieu, 75252 Paris \\ Cedex 05, France. URA 765 of CNRS
}

(May 13, 2019)

\begin{abstract}
In this Letter we consider a system of $N$ pairwise finite-range interacting atoms and prove rigorously that in the zero-range interaction limit all the eigenstates and eigenenergies of the Hamiltonian converge to those corresponding to $N$ atoms interacting via the Fermi-Huang regularized pseudopotential. Next, we show that the latter eigensystem (if treated exactly) is invariant under a nontrivial transformation of the interaction potential. Finally, we realize that most of the approximate schemes of many-body physics do not exhibit this invariance: we use this property to resolve all inconsistencies of the Hartree-Fock-Bogoliubov variational formalism known so far.
\end{abstract}

The realization of Bose Einstein Condensate [1] has brought an enormous interest in developing new theoretical approaches and refining the existing ones. The meanfield formalism with contact interactions has been shown to provide a powerful tool for analyzing the properties of trapped Bose gases [2]. Unfortunately, the most general variational mean field approach: the Hartree-FockBogoliubov approximation (HFB), is not yet quite satisfactory if used with contact interactions: it exhibits UVdivergencies, inconsistencies with the Hugenholtz-Pines theorem [3], many-body $T$-matrix calculations [4], and even with the very existence of atomic condensates themselves [5]. Several heuristic modifications of the theory were suggested [3,4.4.6,7], showing a good agreement with the experimental data [8]. In this Letter, we propose a novel, quite straightforward way to cure these inconsistencies.

First, we prove rigorously that the regularized FermiHuang pseudo-potential [9] is not just an ansatz, but provides the exact zero-range limit of the many-body observables along with a cancellation of all the UVdivergencies. Second, we introduce a new family of pseudo-potentials parametrized by a free parameter $\Lambda$ (so-called $\Lambda$-potentials): no exact (after the zero-range approximation has been made) observable depends on it, while some approximate treatments differ for different $\Lambda$. The above conclusions are general and they do not rely on any particular approximation. Finally, as an application of this new potential, we find a particular value for $\Lambda$ such that HFB equations are entirely free of all inconsistencies known so far.

An explicit expression for the $\Lambda$-potential reads

$$
\hat{V}^{\Lambda}(\vec{r})=g_{\Lambda} \delta(\vec{r})\left[\partial_{r}+\Lambda\right](r \cdot) \quad \text { with } \quad g_{\Lambda}=\frac{g_{0}}{1-a \Lambda}
$$

where $\vec{r}=\vec{r}_{1}-\vec{r}_{2}$ is the relative coordinate of two atoms, $a$ is the $s$-wave scattering length, $g_{0}=2 \pi \hbar^{2} a / \tilde{\mu}$ is the usual effective coupling constant and $\tilde{\mu}=m / 2$ is the reduced mass. When $\Lambda=0, \hat{V}^{\Lambda=0}$ coincides with the Fermi-Huang pseudo-potential. For a $1 / r$-divergent wave-function $\psi(\vec{r})=\tilde{\alpha} / r+\eta(\vec{r})$ [10], the action of the $\Lambda$-potential is

$$
\hat{V}^{\Lambda} \psi(\vec{r})=g_{\Lambda} \delta(\vec{r})[\eta(\overrightarrow{0})+\Lambda \tilde{\alpha}]
$$

For a low-energy two-body body scattering process, the eigenstates of the $\Lambda$-potential coincide with the asymptotic form of the $s$-wave eigenstates of any other interaction potential of a scattering length $a$. However, for energies of the order $\frac{\hbar^{2}}{m a^{2}}$ or higher, the actual finite size structure of the potentials comes into play, and the range of the applicability of the zero-range approximation reduces to the particular case of a zero-energy resonance.

Now, we consider a system of $N$ atoms of mass $m$ and coordinates $\{\mathbf{r}\} \equiv\left\{\vec{r}_{1}, \vec{r}_{2}, \ldots, \vec{r}_{N}\right\}$ interacting via $\hat{V}^{\Lambda}$. The Hamiltonian reads

$$
\hat{\mathcal{H}}^{p \cdot p .}=\sum_{i=1}^{N} \frac{p_{i}^{2}}{2 m}+\sum_{\alpha=1}^{\alpha_{\max }} \hat{V}^{\Lambda}\left(r_{\alpha}\right)
$$

where $\alpha=1, \ldots, \alpha_{\max } \equiv N(N-1) / 2$ labels ordered pairs of atoms $\left(i_{\alpha}, j_{\alpha}\right)$, and $\vec{r}_{\alpha}=\vec{r}_{i_{\alpha}}-\vec{r}_{j_{\alpha}}$ is the relative position of the members of the $\alpha$-th pair. As a direct consequence of Eq.(2), any eigenstate $\Psi(\{\mathbf{r}\})$ of (3) is a solution of an interaction-free Schrödinger equation subject to the following contact conditions for all pair $\alpha$ :

$$
\left.\lim _{r_{\alpha} \rightarrow 0} \frac{\partial}{\partial r_{\alpha}}\right|_{\left\{\mathcal{R}_{\alpha}\right\}} \ln \left[r_{\alpha} \Psi\right]=-\frac{1}{a},
$$

where $\left\{\mathcal{R}_{\alpha}\right\} \equiv\left\{\vec{R}_{\alpha},\left\{\vec{r}_{i} \mid i \neq i_{\alpha}, j_{\alpha}\right\}\right\}$ is a set composed of the coordinate of the center of mass $\vec{R}_{\alpha}=\left(\vec{r}_{i_{\alpha}}+\vec{r}_{j_{\alpha}}\right) / 2$ of the $\alpha$-th pair and all other coordinates not belonging to this pair. Indeed, it is easy to show that the $\delta$ singularities in the action of the Hamiltonian (3) on a many-body state $\Psi(\{\vec{r}\})$ cancel each other if and only if $\Psi$ satisfies the contact conditions (11). Notice that these contact conditions do not depend on $\Lambda$, and thus no exact (after the zero-range approximation has been made) eigenstate does.

Now, we are going to show how regularized pseudopotentials arise in the limit of zero-range interactions. For this purpose, we consider $N$ particles of mass $m$, 
interacting via a potential which belongs to a oneparameter family of square-well potentials: $v^{R}(r)=$ $-v_{0}^{R} \Theta(R-r)$. The depth of the potential $v_{0}^{R}>0$ is chosen in such a way that the scattering length $a$ is the same for all members of the family, and each of them supports the same number of $s$-wave bound states, either one or none depending on the sign of the scattering length 11]. The Hamiltonian of the system reads

$$
\hat{\mathcal{H}}^{R}=\sum_{i=1}^{N} \frac{p_{i}^{2}}{2 m}+\sum_{\alpha=1}^{\alpha_{\max }} v^{R}\left(r_{\alpha}\right) .
$$

We wish to prove the following

STATEMENT.- In the limit of infinitely small potential range, the Green's function of the finite-range-interaction Hamiltonian (1) converges to the Green's function of the pseudo-potential Hamiltonian (3):

$$
\lim _{R \rightarrow 0}\left(E-\hat{\mathcal{H}}^{R}\right)^{-1}=\left(E-\hat{\mathcal{H}}^{p \cdot p \cdot}\right)^{-1} .
$$

Proof. As we saw above (see (4)), all the $\Lambda$-potentials lead to the same eigenstates (and thus the same Green's function): hence, without loss of generality, we can limit our proof to the case of the Fermi-Huang pseudo-potential $\hat{V}^{0}$.

Let us define two operator-valued functions:

$$
\hat{\mathcal{G}}_{\hat{a}}^{E}=(E+i \epsilon-\hat{a})^{-1} \quad, \quad \hat{\mathcal{T}}_{\hat{a}, \hat{b}}^{E}=\left(1-\hat{b} \hat{\mathcal{G}}_{\hat{a}}^{E}\right)^{-1} \hat{b} .
$$

The former is the retarded Green's function at energy $E$ for a Hamiltonian $\hat{a}$. The latter is the $T$-matrix of a perturbation $\hat{b}$ in presence of the background Hamiltonian $\hat{a}$. Two relations will be heavily used in what follows. First is the Lippman-Schwinger relation between the Green's function of the "full Hamiltonian" $\hat{a}+\hat{b}$ and the one of the background:

$$
\hat{\mathcal{G}}_{\hat{a}+\hat{b}}^{E}=\hat{\mathcal{G}}_{\hat{a}}^{E}+\hat{\mathcal{G}}_{\hat{a}}^{E} \hat{\mathcal{T}}_{\hat{a}, \hat{b}}^{E} \hat{\mathcal{G}}_{\hat{a}}^{E} .
$$

The second is the Lupu-Sax formula [12] relating the $T$ matrices of the same perturbation but in two different background Hamiltonians $\hat{a}_{1}$ and $\hat{a}_{2}$ :

$$
\hat{\mathcal{T}}_{\hat{a}_{2}, \hat{b}}^{E}=\left[1-\hat{\mathcal{T}}_{\hat{a}_{1}, \hat{b}}^{E}\left(\hat{\mathcal{G}}_{\hat{a}_{2}}^{E}-\hat{\mathcal{G}}_{\hat{a}_{1}}^{E}\right)\right]^{-1} \hat{\mathcal{T}}_{\hat{a}_{1}, \hat{b}}^{E} .
$$

Introduce also a family of "reduced Hamiltonians" $\hat{\mathcal{H}}_{\{\alpha\}}^{R}$ and a family of reference Hamiltonians $\hat{h}_{\alpha}^{E}$

$\hat{\mathcal{H}}_{\{\alpha\}}^{R}=\sum_{i=1}^{N} \frac{p_{i}^{2}}{2 m}+\sum_{\beta=1}^{\alpha} v^{R}\left(r_{\beta}\right) \quad$ and $\quad \hat{h}_{\alpha}^{E}=\frac{p_{\alpha}^{2}}{2 \tilde{\mu}}+E$,

where $\vec{p}_{\alpha}=\left(\vec{p}_{i_{\alpha}}-\vec{p}_{j_{\alpha}}\right) / 2$ is the relative momentum for the $\alpha$-th pair. Each reference Hamiltonian is just a sum of the relative kinetic energy for the corresponding pair and the energy $E$ at which the Green's functions (6) are compared. The Green's function of the $\alpha$-th reference
Hamiltonian is proportional to the zero-energy Green's function for the relative motion of two particles:

$$
\left\langle\{\mathbf{r}\}\left|\hat{\mathcal{G}}_{\hat{h}_{\alpha}^{E}}^{E}\right|\left\{\mathbf{r}^{\prime}\right\}\right\rangle=-\frac{\tilde{\mu}}{2 \pi \hbar^{2}\left|\vec{r}_{\alpha}-\vec{r}_{\alpha}^{\prime}\right|} \delta\left(\left\{\boldsymbol{\mathcal { R }}_{\alpha}-\boldsymbol{\mathcal { R }}_{\alpha}^{\prime}\right\}\right) .
$$

In turn the $T$-matrix of the interaction potential $\hat{v}_{\alpha}^{R} \equiv$ $v^{R}\left(r_{\alpha}\right)$ in presence of $\hat{h}_{\alpha}^{E}$ can be expressed through the zero-energy two-body T-matrix of it:

$$
\left\langle\{\mathbf{r}\}\left|\hat{\mathcal{T}}_{\hat{h}_{\alpha}^{E}, \hat{v}_{\alpha}^{R}}^{E}\right|\left\{\mathbf{r}^{\prime}\right\}\right\rangle=g_{0} D^{R}\left(\vec{r}_{\alpha}, \vec{r}_{\alpha}^{\prime}\right) \delta\left(\left\{\boldsymbol{\mathcal { R }}_{\alpha}-\boldsymbol{\mathcal { R }}_{\alpha}^{\prime}\right\}\right) .
$$

The kernel $D^{R}$ is zero when $r_{\alpha}>R$ or $r_{\alpha}^{\prime}>R$ and is normalized to unity as $\int d^{3} \vec{r} d^{3} \vec{r}^{\prime} D^{R}\left(\vec{r}, \vec{r}^{\prime}\right)=1$. An explicit expression for it can be found in Ref. 13]. In the limit of zero-range interaction, the kernel obviously converges to a product of delta-functions, and hence the $T$-matrix converges to:

$$
\hat{\mathcal{T}}_{\hat{h}_{\alpha}^{E}, \hat{v}_{\alpha}^{R}}^{E} \stackrel{R \rightarrow 0}{\longrightarrow} g_{0} \delta\left(\vec{r}_{\alpha}\right) .
$$

Notice that by construction of the reference Hamiltonian $\hat{h}_{\alpha}^{E}$ neither the Green's function $\hat{\mathcal{G}}_{\hat{h}_{\alpha}^{E}}^{E}$ nor the $T$-matrix $\hat{\mathcal{T}}_{\hat{h}_{\alpha}^{E}, \hat{v}_{\alpha}^{R}}^{E}$ depend on energy $E$.

Using relations (8, 9), the full many-body Green's function of the system can be rigorously expressed through the zero-energy two-body $T$-matrices (12) of the interaction potential $v^{R}(r)$. Removing from the Hamiltonian (5) one pair interaction after another, we obtain the following chain relation:

$$
\begin{aligned}
& \left(E-\hat{\mathcal{H}}^{R}\right)^{-1} \equiv \hat{\mathcal{G}}_{\hat{\mathcal{H}}^{R}}^{E} \equiv \hat{\mathcal{G}}_{\mathcal{\mathcal { H }}_{\{\alpha \max \}}^{R}}^{E} \\
& \uparrow \ldots \\
& \hat{\mathcal{G}}_{\hat{\mathcal{H}}_{\{\alpha\}}^{R}}^{E}=\hat{\mathcal{G}}_{\hat{\mathcal{H}}_{\{\alpha-1\}}^{R}}^{E} \\
& +\hat{\mathcal{G}}_{\mathcal{\mathcal { H }}_{\{\alpha-1\}}^{R}}^{E}\left[1-\hat{\mathcal{T}}_{\hat{h}_{\alpha}^{E}, \hat{v}_{\alpha}^{R}}^{E}\left(\hat{\mathcal{G}}_{\hat{\mathcal{H}}_{\{\alpha-1\}}^{R}}^{E}-\hat{\mathcal{G}}_{\hat{h}_{\alpha}^{E}}^{E}\right)\right]^{-1} \\
& \times \hat{\mathcal{T}}_{\hat{h}_{\alpha}^{E}, \hat{v}_{\alpha}^{R}}^{E} \hat{\mathcal{G}}_{\hat{\mathcal{H}}_{\{\alpha-1\}}^{R}}^{E} \\
& \uparrow \ldots \\
& \hat{\mathcal{G}}_{\hat{\mathcal{H}}_{\{0\}}^{E}}^{E} \equiv\left(E-\sum_{i=1}^{N} \frac{p_{i}^{2}}{2 m}\right)^{-1} .
\end{aligned}
$$

This relation is the cornerstone of the proof.

Imagine now that the full Green's function $\hat{\mathcal{G}}_{\hat{\mathcal{H}}^{R}}^{E}$ acts on a state $|\Psi\rangle$ whose wave function $\Psi(\{\mathbf{r}\})$ is regular everywhere. Computation of the result of this action $\left\langle\{\mathbf{r}\}\left|\hat{\mathcal{G}}_{\hat{\mathcal{H}}^{R}}^{E}\right| \Psi\right\rangle$ involves expressions of a form $\hat{\mathcal{T}}_{\hat{h}_{\alpha}^{E}, \hat{v}_{\alpha}^{R}}^{E}\left(\hat{\mathcal{G}}_{\hat{\mathcal{H}}_{\{\alpha-1\}}^{R}}^{E}-\hat{\mathcal{G}}_{\hat{h}_{\alpha}^{E}}^{E}\right) \hat{\mathcal{T}}_{\hat{h}_{\alpha}^{E}, \hat{v}_{\alpha}^{R}}^{E} \Xi$ where $\Xi(\{\mathbf{r}\})$ is a regular function, and in the limit $R \rightarrow 0$, leads to the expressions of the following type:

$$
g_{0} \delta\left(\vec{r}_{\alpha}\right)\left[\Gamma\left(\vec{r}_{\alpha}\right)-\frac{\tilde{\alpha}}{r_{\alpha}}\right] .
$$


(In Eq.(15), both $\Gamma\left(\vec{r}_{\alpha}\right)$ and $\tilde{\alpha}$ depend also on $\left\{\boldsymbol{\mathcal { R }}_{\alpha}\right\}$.) $\Gamma$ is given by

$$
\Gamma\left(\vec{r}_{\alpha}\right)=\int d^{3 N}\left\{\mathbf{r}^{\prime}\right\}\left\langle\{\mathbf{r}\}\left|\hat{\mathcal{G}}_{\hat{\mathcal{H}}_{\{\alpha-1\}}^{R \rightarrow 0}}^{E}\right|\left\{\mathbf{r}^{\prime}\right\}\right\rangle g_{0} \delta\left(\vec{r}_{\alpha}^{\prime}\right) \Xi\left(\left\{\mathbf{r}^{\prime}\right\}\right),
$$

and $\tilde{\alpha}=-\left.g_{0}\left(\tilde{\mu} / 2 \pi \hbar^{2}\right) \Xi(\{\mathbf{r}\})\right|_{\vec{r}_{\alpha}=0}$. Now using the definition of the Green's function $\hat{\mathcal{G}}_{\hat{\mathcal{H}}_{\{\alpha-1\}}^{R}}^{E}$, we find that $\Gamma\left(\vec{r}_{\alpha}\right)$ has an UV singularity of form $\tilde{\alpha} / r_{\alpha}$ (the same as the second term in the expression (15) has) (see 14]). This leads to

$$
g_{0} \delta\left(\vec{r}_{\alpha}\right)\left[\Gamma\left(\vec{r}_{\alpha}\right)-\frac{\tilde{\alpha}}{r_{\alpha}}\right]=\hat{V}_{\alpha}^{0} \Gamma\left(\vec{r}_{\alpha}\right)
$$

i.e. the expression (15) involves the Fermi-Huang pseudo-potential $\hat{V}_{\alpha}^{0} \equiv V^{0}\left(r_{\alpha}\right)$ (c.f. (2) at $\Lambda=0$ ). This justifies the following limit:

$$
\hat{\mathcal{T}}_{\hat{h}_{\alpha}^{E}, \hat{v}_{\alpha}^{R}}^{E}\left(\hat{\mathcal{G}}_{\hat{\mathcal{H}}_{\{\alpha-1\}}^{R}}^{E}-\hat{\mathcal{G}}_{\hat{h}_{\alpha}^{E}}^{E}\right) \stackrel{R \rightarrow 0}{\rightarrow} \hat{V}_{\alpha}^{0} \hat{\mathcal{G}}_{\hat{\mathcal{H}}_{\{\alpha-1\}}^{R \rightarrow 0}}^{E} .
$$

Inserting the above substitution at every level of the chain procedure (14) and collecting all the terms [15] one finally arrives at $\lim _{R \rightarrow 0} \hat{\mathcal{G}}_{\hat{\mathcal{H}}^{R}}^{E}=\hat{\mathcal{G}}_{\hat{\mathcal{H}}^{p . p .}}^{E}$, Q.E.D..

Notice that the relation (17) clearly shows that the role of the regularizing operator in the pseudo-potential expression (1) is to subtract the free propagators $\hat{\mathcal{G}}_{\hat{h}_{\alpha}^{E}}^{E}$ already taken into account by the two-body $T$-matrix $\hat{\mathcal{T}}_{\hat{h}_{\alpha}^{E}, \hat{v}_{\alpha}^{R}}^{E}$. As a result, UV divergencies disappear at each level of the chain recursion (14).

As an application of the $\Lambda$-potential, we consider now the HFB theory for $\mathrm{N}$ bosons interacting via $V^{\Lambda}$ with $a>0$, in a box of a size $L$. As we will see, the $\Lambda$-freedom in choosing the effective Hamiltonian (3) offers the following advantages: (a) Unlike for the conventional HFB formalism $(\Lambda=0)$, there exists a range of $\Lambda$ such that the atomic condensate constitutes the minimum of the HFB functional in the low density regime [16]; (b) For a particular value, $\Lambda=\Lambda^{\star}$, HFB equations are consistent with the results of the ladder approximation for the many-body $T$-matrix [4] and the Hugenholtz-Pines theorem is satisfied; (c) In the vicinity of $\Lambda^{\star}$ the ground state energy of the system is consistent with Bogoliubov's predictions.

The HFB approximation is twofold. First, it breaks the $U(1)$ symmetry: the atomic field $\hat{\psi}$ is split into a classical field $\Phi$ and a quantum fluctuation $\hat{\phi}=\hat{\psi}-\Phi$. Second, the exact density operator is replaced by a Gaussian variational ansatz: $\hat{D} \equiv \exp \left(-\hat{K} / k_{\mathrm{B}} T\right) / Z$, where $Z$ is the partition function and the quadratic variational Hamiltonian is

$$
\begin{aligned}
\hat{K}[h, \Delta, \Phi]= & \frac{1}{2} \iint d^{3} \overrightarrow{r_{1}} d^{3} \overrightarrow{r_{2}}\left[\hat{\phi}^{\dagger}\left(\overrightarrow{r_{1}}\right) h\left(\overrightarrow{r_{1}}, \overrightarrow{r_{2}}\right) \hat{\phi}\left(\vec{r}_{2}\right)\right. \\
& \left.+\hat{\phi}^{\dagger}\left(\vec{r}_{1}\right) \Delta\left(\vec{r}_{1}, \vec{r}_{2}\right) \hat{\phi}^{\dagger}\left(\vec{r}_{2}\right)+\text { h.c. }\right] .
\end{aligned}
$$

For what follows, we introduce the coordinates $\vec{R}=\left(\vec{r}_{1}+\right.$ $\left.\vec{r}_{2}\right) / 2$ and $\vec{r}=\vec{r}_{1}-\vec{r}_{2}$. The second-quantized form of the full Hamiltonian (3) reads

$$
\hat{\mathcal{H}}^{\Lambda}=\int d^{3} \vec{R}\left\{\hat{\psi}^{\dagger}\left(-\frac{\hbar^{2}}{2 m} \Delta\right) \hat{\psi}+\frac{g_{\Lambda}}{2} \hat{\psi}^{\dagger} \hat{\psi}^{\dagger} \hat{\hat{\psi}} \hat{\psi}\right\},
$$

where

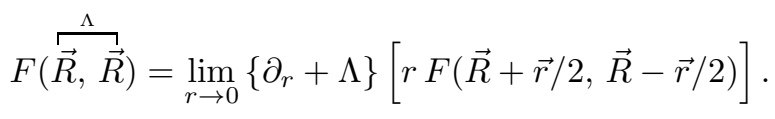

is a shortened notation for the action of the regularizing operator (see (11)). Using Wick's theorem, we obtain an approximate grand canonical potential $J \equiv$ $E^{\Lambda}-\mu N-T S$, where $E^{\Lambda}=\operatorname{Tr}\left[\hat{\mathcal{H}}^{\Lambda} \hat{D}\right]$ is the energy, $N$ is the number of particles, and $S=-k_{\mathrm{B}} \operatorname{Tr}[\ln (\hat{D}) \hat{D}]$ is the entropy. Minimization of $J$ with respect to the three variational fields $h, \Delta$ and $\Phi$ leads to the following implicit equations for these fields:

$$
\begin{aligned}
h\left(\overrightarrow{r_{1}}, \overrightarrow{r_{2}}\right) & =-\frac{\hbar^{2}}{2 m}\left(\vec{\nabla}^{2} \delta\right)(\vec{r})+\left[\hbar \Sigma_{11}^{\Lambda}-\mu\right] \delta(\vec{r}) \\
\Delta\left(\vec{r}_{1}, \vec{r}_{2}\right) & =\hbar \Sigma_{12}^{\Lambda} \delta(\vec{r}) \\
-\frac{\hbar^{2}}{2 m} \Delta \Phi & +\left[g_{\Lambda}\left(2 \tilde{n}+|\Phi|^{2}\right)-\mu\right] \Phi+g_{\Lambda} \tilde{\kappa}_{\Lambda} \Phi^{*}=0,
\end{aligned}
$$

where $\hbar \Sigma_{11}^{\Lambda}=2 n g_{\Lambda}$ and $\hbar \Sigma_{12}^{\Lambda}=g_{\Lambda}\left(\Phi^{2}+\tilde{\kappa}_{\Lambda}\right)$ are the selfenergies, $\tilde{n}=\operatorname{Tr}\left[\hat{\phi}^{\dagger}(\vec{R}) \hat{\phi}(\vec{R}) \hat{D}\right]$ is the non-condensed density, $n=|\Phi|^{2}+\tilde{n}$ is the total density, and $\tilde{\kappa}_{\Lambda}=\tilde{\kappa}(\overrightarrow{\vec{R}, \vec{R}})$ results from the action of the regularizing operator (20) on the anomalous density $\tilde{\kappa}\left(\vec{r}_{1}, \vec{r}_{2}\right)=\operatorname{Tr}\left[\hat{\phi}\left(\vec{r}_{1}\right) \hat{\phi}\left(\vec{r}_{2}\right) \hat{D}\right]$.

The diagonalization of the variational Hamiltonian $\hat{K}$ leads to the following quasi-particle spectrum:

$$
\hbar \omega_{k}=\left(\frac{\hbar^{2} k^{2}}{2 m}+2 g_{\Lambda} \Phi^{2}\right)^{\frac{1}{2}}\left(\frac{\hbar^{2} k^{2}}{2 m}-2 g_{\Lambda} \tilde{\kappa}_{\Lambda}\right)^{\frac{1}{2}} .
$$

Eqs.(21,22) clearly show that HFB is $\Lambda$-dependent. As it has been shown in Ref. [4], this approach is only able to provide a Born approximation for the diagonal selfenergy $\hbar \Sigma_{11}$, hence its explicit $\Lambda$-dependence. However, this is not the case for $\hbar \Sigma_{12}$; indeed, the total pairing field reproduces the contact conditions (四) of a two-body wave function

$$
\left\langle\hat{\psi}\left(\vec{r}_{1}\right) \hat{\psi}\left(\vec{r}_{2}\right)\right\rangle=\left(\Phi^{2}+\tilde{\kappa}_{0}\right)\left(1-\frac{a}{r}\right)+\mathcal{O}(r),
$$

and as a result $\hbar \Sigma_{12}^{\Lambda}=g_{0}\left(\Phi^{2}+\tilde{\kappa}_{0}\right)$ for all $\Lambda$.

Requiring that all the eigen-energies (22) are real, we find that for zero temperature and densities below a value of $n_{\text {crit }}=\frac{\pi}{192 a^{3}}$ the existence of an atomic condensate $(\Phi \neq 0)$ implies the following constraint on $\Lambda$ : 


$$
\Lambda^{\star} a \leq \Lambda a<1 \quad \text { with } \quad \Lambda^{\star} a=\frac{\tilde{\kappa}_{0}}{\Phi^{2}+\tilde{\kappa_{0}}} .
$$

At the lower limit $\Lambda=\Lambda^{\star}$, the $\Lambda$-regularized anomalous density disappears, and the theory become fully consistent with the results of the many-body $T$-matrix calculations in the ladder diagrams approximation [4]

$$
\begin{aligned}
& \tilde{\kappa}_{\Lambda^{\star}}=0 \quad ; \quad \hbar \Sigma_{11}^{\Lambda^{\star}}=2 n g_{\Lambda^{\star}} \quad ; \quad \hbar \Sigma_{12}^{\Lambda^{\star}}=g_{\Lambda^{\star}} \Phi^{2} \\
& g_{\Lambda^{\star}}=g_{0}\left[1+\frac{\tilde{\kappa}_{0}}{\Phi^{2}}\right]=T^{\mathrm{MB}}(\overrightarrow{0}, \overrightarrow{0}, \overrightarrow{0} ; 0),
\end{aligned}
$$

yielding a gapless spectrum [17,18].

Consider now the zero-temperature low-density limit of our equations. Assuming $\Lambda a$ to be of the order of $\sqrt{n a^{3}}$ and neglecting all the terms of order $n a^{3}$ or higher, the energy $E^{\Lambda}$ is independent of $\Lambda$ and coincides with the well known Bogoliubov's result

$$
E^{\Lambda}=\frac{g_{0}}{2} n N\left(1+\frac{128}{15 \sqrt{\pi}} \sqrt{n a^{3}}+\ldots\right) .
$$

The $\Lambda$-potential based variational HFB model is therefore consistent with the perturbative Bogoliubov's approach. As the density increases the parameter $\Lambda^{\star}$ increases as well, and at a critical density $n_{\text {crit }}=\frac{\pi}{192 a^{3}}$ we find $\Lambda^{\star} a=1$ : the energy diverges and the mean field treatment breaks down.

Note in conclusion that the $\Lambda$-invariance described in our Letter holds even if the constant $\Lambda$ is replaced by an arbitrary field $\Lambda(\vec{R})$. The generalization of our HFB theory to the case of the trapped gases is thus straightforward: One has simply to fix $\Lambda$ as $\Lambda(\vec{R})=$ $\Lambda^{\star}\left(\tilde{\kappa}_{0}(\vec{R}), \Phi^{2}(\vec{R})\right)$ according to $(24)$ at every point $\vec{R}$ of the trap.

As a extension of this work, we mention that using a procedure similar to the $3 \mathrm{D}$ case, it is possible to obtain the low-dimensional analogs of the $\Lambda$-potential:

$$
\begin{aligned}
& V_{2 \mathrm{D}}^{\Lambda}(\vec{\rho})=-\frac{\pi \hbar^{2}}{\tilde{\mu}} \frac{1}{\log (q \Lambda R)} \delta(\vec{\rho})\left\{1-\log (q \Lambda \rho) \rho \frac{\partial}{\partial \rho}\right\} \\
& V_{1 \mathrm{D}}^{\Lambda}(z)=-\frac{\hbar^{2}}{\tilde{\mu}} \frac{\Lambda}{\Lambda a_{1 \mathrm{D}}-1} \delta(z)\left\{1+\frac{1}{2 \Lambda} \frac{\partial}{\partial z}\left(\left.\left.\right|_{0+}\right|_{0-}\right)\right\},
\end{aligned}
$$

where $q=e^{C} / 2, C$ is the Euler's constant, $R$ is the $2 \mathrm{D}$ effective hard disk radius and $a_{1 \mathrm{D}}$ is the $1 \mathrm{D}$ scattering length 19,20].

Acknowledgments. Authors are grateful to A. LupuSax, R. Shakeshaft, R. Thompson, and especially to Y. Castin for enlightening discussions on the subject. This work was supported by the NSF grant PHY0070333.

(a) email: olshanii@physics.usc.edu (b) email: pricoupenko@lptl.jussieu.fr

[1] M.H. Anderson, J.R. Ensher, M.R. Matthews, C.E. Wieman, and E.A. Cornell, Science 269, 198 (1995); K.B. Davis, M.O. Mewes, M.R. Andrews, N.J. van Druten, D.S. Durfee, D.M. Kurn, and W. Ketterle, Phys. Rev. Lett. 75, 3969 (1995); C.C. Bradley, C.A. Sackett, and R.G. Hulet, Phys. Rev. Lett. 78, 985 (1997).

[2] F. Dalfovo, S. Giorgini, L.P. Pitaesvkii and S. Stringari, Reviews of Modern Physics 71, 463 (1999).

[3] A. Griffin, Phys. Rev. B 53, 9341 (1996).

[4] M. Bijlsma and H. T. C. Stoof, Phys. Rev. A 55, 498 (1997).

[5] L. Pricoupenko, cond-mat/0006263

[6] D.A.W. Hutchinson, R.J. Dodd and K. Burnett, Phys. Rev. Lett. 81,2198 (1998).

[7] S. Giorgini, Phys. Rev. A 61, 63615 (2000).

[8] D.S. Jin, M.R. Matthews, J.R. Ensher, C.E. Wieman, and E.A. Cornell, Phys. Rev. Lett. 78, 764 (1997); D.M. Stamper-Kurn, H.-J. Miesner, S. Inouye, M.R. Andrews, and W. Ketterle, Phys. Rev. Lett. 81, 500 (1998).

[9] K. Huang, Statistical Mechanics (Wiley, NY, 1987).

[10] $\eta(\vec{r})$ is a regular function and $\eta(\overrightarrow{0})=\lim _{r \rightarrow 0}\left[\psi(\vec{r})-\frac{\tilde{\alpha}}{r}\right] \quad$, is the regular part of the wavefunction.

[11] $v_{0}$ is the smallest positive solution of the implicit equation $a=R\left(1-\tan \left(k_{0} R\right) /\left(k_{0} R\right)\right)$, where $k_{0}=\sqrt{m v_{0}} / \hbar$.

[12] Adam Lupu-Sax, Quantum Scattering Theory and Applications, PhD Thesis, Harvard University (1998).

[13] H. Cheng, E. Vilallonga, H. Rabitz, Phys. Rev. A, 42, 5232 (1990).

[14] As an intermediate step of the proof one should show that $\Delta_{\vec{r}_{\alpha}} \Gamma\left(\vec{r}_{\alpha}\right)=-4 \pi \tilde{\alpha} \delta\left(\vec{r}_{\alpha}\right)+$ regular terms

[15] This calculation also involves expressions of a form $\hat{\mathcal{T}}_{\hat{h}_{\alpha}^{E}, \hat{v}_{\alpha}^{R}}^{E} \Xi$ where the limit $\hat{\mathcal{T}}_{\hat{h}_{\alpha}^{E}, \hat{v}_{\alpha}^{R}}^{E} \Xi \stackrel{R \rightarrow 0}{\rightarrow} \hat{V}_{\alpha}^{0} \Xi$ is also well justified.

[16] The ground state of a real many-body Hamiltonian is not an atomic condensate, due to the presence of twobody bound states. For $a>0$ the pseudo-potential also supports a bound-state which coincides with the asymptotic form of the highest $s$-wave bound state of the real two-body potential. As a result, at $\Lambda=0$ (and more generally at $\Lambda<\Lambda^{\star}$ ) HFB describes a molecular condensate, instead of the atomic one [5].

[17] In short, at $\Lambda=\Lambda^{\star}$ our theory justifies the G2-type mean-field model, due to the classification scheme given in N.P. Proukakis, S.A. Morgan, S. Choi, and K. Burnett, Phys. Rev. A, 58, 2435 (1998).

[18] We do not consider here the effect of the "lost mode" which appears for a zero gap spectrum - M. Lewenstein and L. You, Phys. Rev. Lett. 773489 (1996). We assume that $\Lambda$ can be chosen arbitrarily close to $\Lambda^{\star}$, so that an infinitely small gap remains.

[19] The coefficients in front of the potentials are the lowenergy two-body $T$-matrices $T^{2 B}\left(\overrightarrow{0}, \overrightarrow{0} ;-\hbar^{2} \Lambda^{2} / 2 \tilde{\mu}\right)-$ H.T.C. Stoof, L.P.H. de Goey, W.M.H.M. Rovers, P.S.M. Kop Jansen, and B.J. Verhaar, Phys. Rev. A 38, 1248 (1988).

[20] A particular member of the 2D family can be found in K. Wódkiewicz, Phys. Rev. A 43, 68 (1991). The usual $1 \mathrm{D} \delta$-potential $g_{1 \mathrm{D}} \delta(z)$ with $g_{1 \mathrm{D}}=-\hbar^{2} / \tilde{\mu} a_{1 \mathrm{D}}$ constitutes 
the $\Lambda=+\infty$ member of the 1D family. 